\title{
Reaching Within a Dynamic Virtual Environment
}

\author{
Assaf Y. Dvorkin, Robert V. Kenyon, and Emily A. Keshner
}

\begin{abstract}
Planning and execution of reaching movements requires a series of computational processes that involves the localization of both the target and initial arm position, and the translation of this spatial information into appropriate motor commands that will bring the hand to the target. Voluntary and/or involuntary changes in the spatial relationship between our hand and the space in which we plan to reach may occur at any time. In the current study we investigated the effects of shifting both the central and peripheral visual field on visuomotor control using a virtual visual environment. In two separate experiments, six seated healthy subjects were exposed to either stationary or roll motion of a $3 D$ virtual scene while reaching for a visual target that remained stationary in space or unexpectedly shifted to a second position with different inter-stimulus intervals. Our initial results suggest that both temporal and spatial aspects of the reaching movement were affected by roll of the visual field. Whereas the results indicate that subjects were able to amend their ongoing motion to match the target position with both scene conditions, the presence of peripheral visual field motion produced significantly longer pauses during the reach movement when the target shifted in space. We conclude from this that motion in the peripheral field of view interfered with the ability to simultaneously process two consecutive stimuli. The terminal arm posture also exhibited a drift in the direction opposite to the roll motion suggesting that subjects were compensating for a perceived change in their visual reference frame.
\end{abstract}

\section{INTRODUCTION}

$\mathrm{D}$ URING the execution of a motor task when either we are moving or when the world is moving around us, the central nervous system (CNS) must monitor online body orientation by updating the internal representation of visual space. Such online control is particularly suitable to overcome external perturbations likely to impair movement accuracy. An experimental paradigm which has been extensively used to probe the online responses of the CNS to external perturbations is called the 'double-step target displacement' paradigm. This paradigm consists of changing the spatial goal of the movement by unexpectedly changing the location of a visual target either before or following movement initiation [1]-[3]. Studies have shown that both young and elderly healthy subjects are able to amend their

Manuscript received April 1, 2006. This work was supported by NIHNIDCD grant DC05235.

A. Y. Dvorkin is with the Sensory Motor Performance Program, Rehabilitation Institute of Chicago, Chicago, IL 60611 USA (phone: 312238-5828; fax: 312-238-2208; e-mail: a-dvorkin@northwestern.edu).

R. V. Kenyon, is with the Department of Computer Science, University of Illinois at Chicago, Chicago, IL 60607 USA (e-mail: kenyon@uic.edu).

E. A. Keshner is with the Sensory Motor Performance Program, Rehabilitation Institute of Chicago, and the Department of Physical Medicine and Rehabilitation, Feinberg School of Medicine, Northwestern University, Chicago, IL 60611 USA (e-mail: eak@northwestern.edu). ongoing movements in response to the unexpected target displacement [2], [4]. However, these movements have only been tested in stationary visual environments so that the subjects do not need to modify their programming of the visual space. During most active motions (e.g., reaching for your coffee cup while driving) the external world is moving at the same time.

There is ample evidence that dynamic visual inputs affect motor behavior. A wide variety of moving visual stimuli have been employed to study this issue such as tilting or rotating rooms or projected displays simulating a moving visual scene, e.g., [5], [6]. For example, studies with visual field motion have demonstrated an increase in postural instability [7], with robust postural changes in the roll and pitch planes [8]. Furthermore, it has been shown that subjects who preformed a reaching task while sitting in a rotating chamber made errors when pointing to targets. By contrast, reaching movements made during natural, voluntary torso rotation seem to be accurate [5]. Finally, visual motion has been also shown to affect locomotion during stepping tests, when subjects were exposed to the scene during treadmill walking [9]. Yet, the weighting of visual information and the exact role of visual motion in human motor control is not well understood.

In recent years, virtual reality technology has emerged as a powerful tool to study motor control in healthy subjects and in different populations of patients (e.g., stroke, labyrinthine deficient adults), e.g., [10]-[12]. In our study we examined how dynamic visual field might affect reaching movements. The use of virtual reality technology provides us with a flexible tool, which simulates real world like visual motion, where we can easily and precisely manipulate the scene and virtual objects that appear within it.

In the current study subjects were exposed to either an immersive 3D virtual environment (VE) which was matched to head motion or to roll motion of the VE, while reaching toward visual targets using the double-step paradigm in a $3 \mathrm{D}$ space. The main goal of this study was to investigate the effect of visual motion on planning and execution of reaching movements as well as on the efficiency of online visuomotor adjustments.

\section{MAterials AND MethodS}

\section{A. Subjects}

To date, six young healthy adults (age 25-35 years) participated in the study. All subjects were right-handed and had normal or corrected-to-normal vision. Subjects gave informed consent in accordance with the Institutional 
Review Board of Feinberg School of Medicine, Northwestern University.

\section{B. Apparatus and Data Collection}

Subjects were exposed to an immersive 3D wide field of view VE (scene). The VE was projected via a stereo-capable projector onto a $2.6 \mathrm{~m} \times 3.2 \mathrm{~m}$ back-projection screen. An Electrohome Marquis 8500 projector throws a full-color stereo workstation field $(1024 \times 768$ stereo $)$ at $120 \mathrm{~Hz}$ onto the screen, where the pixels are evenly distributed throughout the scene. The environment consisted of a $30.5 \mathrm{~m}$ wide by $6.1 \mathrm{~m}$ high by $30.5 \mathrm{~m}$ deep room containing round columns with patterned rugs and painted ceiling. Beyond the virtual room was a landscape consisting of mountains, meadows, sky and clouds. Visual targets, which appeared with the scene, were generated as 3D virtual ball-shape target (Fig. 1a). Field sequential stereo images of the environment were separated into right and left eye images using liquid crystal stereo shutter glasses worn by the subject (Crystal Eyes, StereoGraphics Inc.). The correct perspective and stereo projections for the scene were computed using values for the current orientation of the head (6 DOF) supplied at $120 \mathrm{~Hz}$ by reflective markers (Motion Analysis) attached to the stereo shutter glasses. Consequently, virtual

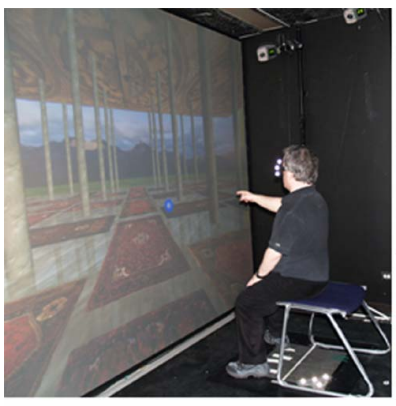

(a)

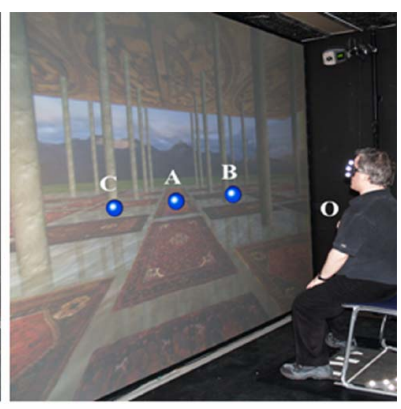

(b)

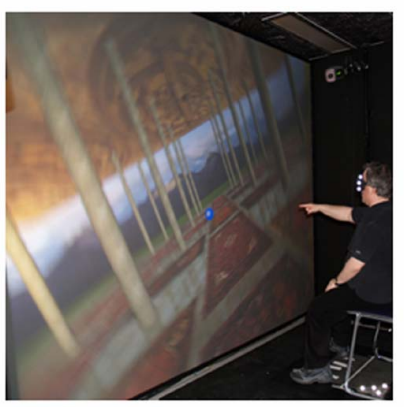

(c)

Fig. 1. (a) Screen shot of an individual performing within the VE. (b) Spatial arrangement of visual targets. Note that the letter labels do not appear within the VE. (c) Screen shot during performance in the roll condition.

objects retained their true perspective and position in space regardless of the subjects' movement.

Hand 3D movements were recorded using a six camera Motion Analysis system (Motion Analysis, Inc.). Reflective markers were attached to the right arm and were tracked at $120 \mathrm{~Hz}$. Commercial software (EVaRT and Matlab) were used to generate and analyze the kinematic data.

\section{Procedures}

All subjects participated in both Experiment 1 and 2. The experiments were randomly presented to minimize order dependencies. Subjects sat comfortably on a stationary stool located at a fixed distance $(1.2 \mathrm{~m})$ from the screen, with their feet in full contact with the floor. Three virtual visual targets appeared with the VE as blue spheres with a $1 \mathrm{~cm}$ radius (defined as A, B and C in Fig. 1b). Sequence and duration of the targets' appearance was controlled. At the start of each experiment, spatial position of each target was defined in terms of the arm length and sternum position of the subject. The central target (A) was located directly in front of the subject at a distance equal to $90 \%$ of arm length and $20 \mathrm{~cm}$ above the sternum position. The other targets were located $15 \mathrm{~cm}$ to the right (B) or left (C) of the central target.

Experiment 1: Each experiment began with 20 practice trials. Five blocks of trials were presented, each containing 12 single-step trials (with configurations $\mathrm{OA}, \mathrm{OB}$ and $\mathrm{OC}$ ) and 24 double-step trials (with configurations $\mathrm{OAB}$ and $\mathrm{OAC}$ ) in random order. A 2 min rest period was given between each block. For half of the trials in each block, subjects were exposed to a visual scene which was matched to head motion. On the other half, the scene rotated in a counter clockwise direction about the line of sight at a constant velocity of $130 \%$ s (Fig. 1c). In total, subjects completed 180 trials.

For all experimental conditions, each trial began with the right index finger extended and placed on the sternum (defined as $\mathrm{O}$ in Fig. 1b). Subjects were instructed to reach toward the visual target as soon as it appeared in the VE. If the target was displaced, the subjects had to move his/her hand towards the new target location. Subjects were also instructed to keep their hand at the final position till the trial ended, (the scene turned black for $2 \mathrm{~s}$ at the end of each trial). No instructions concerning movement speed or accuracy were given.

As seen in Fig. 2, at time $\mathrm{t}=0$, the initial scene appeared. After a random time interval ranging from 1 to $1.5 \mathrm{~s}$, a visual target appeared and the scene remained stationary or started to roll. In the single-step condition, the target remained visible for $2 \mathrm{~s}$. In the double-step condition, following a prespecified inter-stimulus interval (ISI), the location of the target shifted either left or right (i.e., the central target disappeared and simultaneously a second target appeared) and remained in the new position for $2 \mathrm{~s}$. The target was shifted either before movement initiation (ISI $=50$ or 200 $\mathrm{ms}$ ) or during the movement (ISI $=500 \mathrm{~ms}$ ). A total of 40 double-step trials were presented at each ISI.

Experiment 2: This experimental design was the same as in Experiment 1, except for the duration of the target appearance. In the single-step condition, a target appeared for $200 \mathrm{~ms}$. In the double-step condition, a target appeared and, following a pre-specified ISI (200 or $500 \mathrm{~ms})$, the location of the target was shifted to the left or to the right and remained in the new position for only $200 \mathrm{~ms}$. Thus, subjects were instructed to reach toward the remembered 
location of the target. The experiment included 5 blocks, each containing a mixture of 12 single-step trials and 16 double-step trials. In total, subjects completed 140 trials.
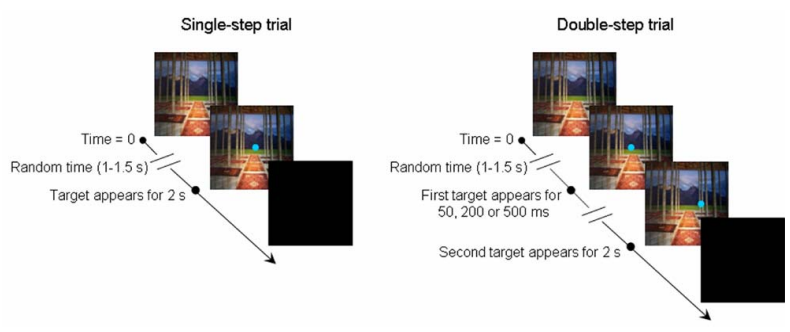

Fig. 2. The double-step paradigm protocol in Experiment 1. Target A and targets A, B are presented for the left and right panels, respectively.

\section{Data Analysis}

Data were low-pass filtered off-line using a $4^{\text {th }}$ order Butterworth digital filter at $8 \mathrm{~Hz}$. A $4 \%$ tangential peak velocity threshold was used to determine the onset and offset of the hand movement. Spatial and temporal kinematic parameters of the reach movement were calculated. These included hand path, reaction time (RT) to movement initiation, movement time (MT) from movement initiation to movement termination, and the duration of any pauses that occurred during the movement.

\section{RESULTS}

Kinematics of the reaching motion within the virtual environment was characterized by similar properties to those described in previous studies in which reaches were executed in the physical world. For the single-step condition, subjects reached toward all single targets without any irregularities of the reach, exhibiting single-peaked bellshaped velocity profiles. For the double-step condition, subjects exhibited double-peaked velocity profiles. Furthermore, three trajectory types were observed: 1) The hand moved directly toward the second target location, 2) the hand moved toward the first target and then changed course during movement, 3 ) the hand moved in between the first and second target locations and changed course during the movement, (Fig. 3a). This shows that subjects were able to amend their ongoing motion in response to target displacement.

Some of the double-step movements contained a pause prior to modifying trajectory direction (Fig. 3b). A pause was defined to be an interval of at least $40 \mathrm{~ms}$ in which the subject's hand was not moving. For all subjects, pause modified movements were only observed for the longest ISI (i.e., $500 \mathrm{~ms}$ ). On average, the proportion of paused movements calculated from the total of double-step movements was lower for Experiment 1 (15.7\%) compared to Experiment 2 (27.7\%). In addition, significantly shorter mean paused movement duration was found for Experiment 1 compared to Experiment 2 (117 vs. $156 \mathrm{~ms} ; \chi_{(1)}^{2}=5.57$, $\mathrm{p}=0.018$ ). Finally, for both the single- and double-step conditions, the $3 \mathrm{D}$ hand path was consistently curved.

\section{A. Effect of Roll Visual Motion}

The analysis on RT and MT did not reveal any significant effect of roll visual motion on the reaching motion, although there was a trend of increasing MT in the presence of roll motion in both experiments. Although the proportion of paused movements was similar in both scene conditions for both experiments, the duration of the pause was only significantly different between the stationary $(120 \mathrm{~ms})$ and rolling $(190 \mathrm{~ms})$ scenes in Experiment $2\left(\chi_{(1)}^{2}=15.8\right.$, $\mathrm{p}<0.0001)$.

Finally, as mentioned earlier, subjects were instructed to keep their hand at the final position until the trial ended. While all subjects kept their hand at the final position for both scene conditions in Experiment 1 (Fig. 3c), an obvious change in hand position was observed during roll in Experiment 2. This was seen for both single- and doublestep trials, where following the main reaching movement the hand continuously moved slowly toward the right which was opposite the direction of the rolling scene (Fig. 3d). (a)

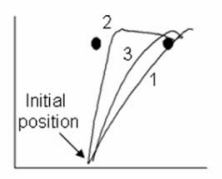

(c)

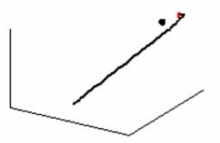

(b)

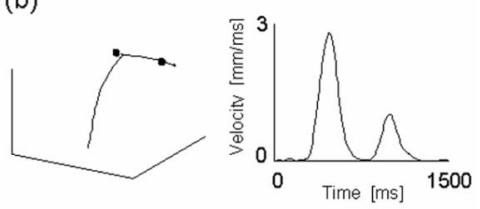

(d)

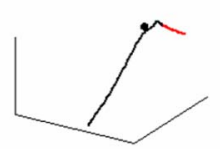

Fig. 3. Examples of (a) different trajectory types (configuration $O A B$ ). A 2D plot of YZ plane, (b) a 3D path and the corresponding tangential velocity profile of a paused movement (configuration OAB). Subject paused for $180 \mathrm{~ms}$, (c) and (d) 3D paths (configuration OB and OA, respectively) showing the main movement (in black) followed by additional movement of the hand (in red). Targets appear as black circles.

\section{DISCUSSION}

The purpose of this study was to evaluate the effect of visual field motion on online visuomotor control of goaldirected arm movements using the double-step paradigm. Subjects were exposed to a visual scene which was matched to head motion and to roll motion of the visual surround, created by a stereo VE projection, while they reached toward virtual targets. Our data indicate that performance in the VE did not differ from that observed in the physical world [4] for both the single- and double-step conditions. Subjects were able to reach toward all targets and to modify their hand trajectory in response to the target displacement. These findings are in agreement with an earlier study [3] which used the double-step task in a stationary VE.

Both temporal and spatial aspects of the reach movement were affected by roll motion of the visual surround, 
particularly when subjects were asked to reach toward a remembered target. Whereas our preliminary results showed a trend in the effect of visual motion on MT, further investigation on a larger population may be necessary to generate statistical significance. In addition, in the presence of roll motion, following the main reaching motion subjects tended to let the hand drift toward the right when the final target disappeared after $200 \mathrm{~ms}$. We surmise that, in the absence of a stable focal image (i.e., the target), subjects were more greatly influenced by the motion of the visual surround and were unable to sustain a constant arm position in space. The arm drifted in the direction opposite of the roll of the visual scene suggesting that subjects were compensating for the perceived change in their visual reference frame.

Previous studies have shown that visual motion of the surround strongly influences the postural response of subjects, e.g., [8]. It is believed that posture is altered because the perception of body orientation in space is shifted by a conflict between the signals from the visual, vestibular and somatosensory systems. Our subjects, however, did not exhibit a tilt of the body during exposure to the roll motion. This was an expected result because our subjects were sitting on a stool during the experiment. We infer that the more stable seated posture and solid support surface inputs from the stool could be more heavily weighted than the sensory conflict arising from roll motion of the visual field. But the drift of the arm with motion of the visual surround could be indicative a perceived shift in visual reference frame and suggests that our subjects were relying more on visual information for localization of the target in space.

Our other significant finding of increased pause duration during the double-step reach to a remembered target when combined with roll motion of the surround was striking. Earlier investigations on the double-step reaching task have suggested that the initial trajectory plan toward the first target continues unmodified until its intended completion and is vectorially added to a second trajectory plan (between an intermediate location and the final target), to yield the plan for the entire modified trajectory [2]. Paused movements have been demonstrated in previous reaching studies [4], [13] and are suggestive of difficulty in performing online trajectory modification. The fact that the mean pause duration increased for the roll condition, implies that visual motion interferes with the ability to prepare in parallel motor responses to the two consecutive visual targets.

In summary, these initial results demonstrate that motion of the visual field affected both planning and execution of the reaching movement, particularly while reaching toward a remembered target. Findings from this ongoing study might have ramifications for both elderly subjects and labyrinthine deficient individuals who have been shown to experience postrotation disturbances of posture, gait and arm movement control [14]. Reaching within a moving visual environment involves complex sensorimotor transformations as a result of the continuous change in the visual reference frame. The posterior parietal cortex (PPC) is assumed to be a critical cortical region involved in the computation and recomputation of the necessary sensorimotor transformations that allow the online hand trajectory adjustments [15]. In fact, an emerging body of work has demonstrated disruption in the online correction of limb movement in patients with parietal damage and in normal subjects receiving transcranial magnetic stimulation to parietal cortex, especially PPC [4], [15], [16]. It might be that PPC is also involved in the control of goal-directed movements performed within a moving visual environment. Knowledge of how visual motion affects both reaching and postural control could eventually lead to developing rehabilitation paradigms that could minimize the disturbing effect of visual motion on motor planning and execution.

\section{ACKNOWLEDGMENT}

We thank Jake Streepey and Leo Wu for their assistance and helpful discussions. We gratefully acknowledge VRCO for supplying CAVE library software.

\section{REFERENCES}

[1] A. Farnè et al., "Visuo-motor control of the ipsilateral hand: Evidence from right brain-damaged patients," Neuropsychologia, vol. 41, pp. 739-757, 2003.

[2] E.A. Henis and T. Flash, "Mechanisms underlying the generation of averaged modified trajectories," Biological Cybernetics, vol. 72, pp. 407-419, 1995.

[3] O. Martin, B. Julian, L. Boissieux, J.D. Gascuel and C. Prablanc, "Evaluating online control of goal-directed arm movement while standing in virtual visual environment," J. Visual Comput Animat, vol. 14, pp. 253-260, 2003.

[4] A.Y. Dvorkin, "Space representation using multiple reference frames in the motor system," Ph.D. dissertation, Hebrew University of Jerusalem, Israel, 2004.

[5] J.V. Cohn, P. DiZio and J.R. Lackner, "Reaching during virtual rotation: Context specific compensations for expected coriolis forces," J. Neurophysiol, vol. 83, pp. 3230-3240, 2000.

[6] J.W. Streepey, R.V. Kenyon and E.A. Keshner, "Field of view and base of support width influence postural responses to visual stimuli during quiet stance," Gait \& Posture, in press.

[7] E.A. Keshner, R.V. Kenyon and J. Langston, "Postural responses exhibit multisensory dependencies with discordant visual and support surface motion," J. Vestibular Research, vol. 14, pp. 307-319, 2004.

[8] F.H. Previc, "The effects of dynamic visual stimulation on perception and motor control," J. Vestibular Research, vol. 2, pp. 285-295, 1992.

[9] Y. Nomura, A.P. Mulavara, J.T. Richards, R. Brady and J.J. Bloomberg, "Optic flow dominates visual scene polarity in causing adaptive modification of locomotor trajectory," Cognitive Brain Research, vol. 25, pp. 624-631, 2005.

[10] A.Y. Dvorkin, M. Shahar and P.L. Weiss, "Reaching within videocapture virtual reality: Using VR as a motor control paradigm," Cyberpsychology \& Behavior, vol. 9, pp. 133-136, 2006.

[11] E.A. Keshner and R.V. Kenyon, "Using immersive technology for postural research and rehabilitation," Assistive Technology, vol. 16, pp. 54-62, 2004.

[12] A. Viau, A.G. Feldman, B.J. McFadyen and M.F. Levin, "Reaching in reality and virtual reality: a comparison of movement kinematics in healthy subjects and in adults with hemiparesis," J. NeuroEng Rehab, vol. $1: 11,2004$.

[13] M. Plotnik, T. Flash, R. Inzelberg, E. Schechtman and A.D. Korczyn, "Motor switching abilities in Parkinson's disease and old age: temporal aspects," J. Neurol Neurosurg Psychiatry, vol. 65, pp. 328$337,1998$. 
[14] J.R. Lackner and P. DiZio, "Vestibular, proprioceptive, and haptic contributions to spatial orientation," Anпи Rev Psychol, vol. 56, pp. 115-147, 2005.

[15] L. Pisella et al., "An 'automatic pilot' for the hand in human posterior parietal cortex: toward reinterpreting optic ataxia," Nature
Neuroscience, vol. 3, pp. 729-736, 2000.

[16] M. Desmurget et al., "Role of the posterior parietal cortex in updating reaching movements to a visual target," Nature Neuroscience, vol. 2, pp. 563-567, 1999. 